\title{
Pre- and postmelting of cadmium telluride
}

\author{
L.P. Shcherbak, P.I. Feichouk, Yu.A. Plevachouk*, O.V. Kopach, L.T. Turyanska \\ Chernivtsi Yu.Fed'kovich State University, Chernivtsi, Ukraine \\ tel.: 380(37) 2298485; E-mail: petro@sacura.chernovtsy.ua \\ "Institute for Applied Physics, Lviv I. Franko State University, Lviv, Ukraine
}

\begin{abstract}
A stepwise character of cadmium telluride melting is shown by using differential thermal analysis and conductivity measurements in the 1323 to $1473 \mathrm{~K}$ temperature range. According to the results of differential thermal analysis the parameters of CdTe melting are determined by the premelting processes that are related to defect production in crystal lattice. The crystallization processes are controlled with the melt state (structure) that depends on its maximum temperature.
\end{abstract}

Keywords: cadmium telluride, postmelting, conductivity, melt structure, polymorphic transformations. Paper received 17.09.99; revised manuscript received 15.12.99; accepted for publication 17.12.99.

\section{Introduction}

In recent years an extensive literature has evolved that indicates at a multistage character of melting for materials with different types (ionic, covalent, metallic) of chemical bonding. Distinct differences exist between stages of premelting, melting, and postmelting [1-5]. The above processes affect the crystallization parameters, as well as structural characteristics of melt-grown crystals. This serves as an evidence that information on features of pre- and postmelting phenomena during interconversions between the contacting condensed phases is of both theoretical and practical value.

One of matters for scientific enquiry that are of theoretical, as well as practical, interest is cadmium telluride. It belongs to a few materials that retain semiconducting properties even at temperatures over that of melting $\left(T_{m}\right)[6,7]$. Unfortunately, the existing information on the structure-sensitive properties of CdTe near $T_{m}$ is few in number and contradictory. Further still, when systematizing the literature data on the high-temperature physico-chemical parameters of CdTe [8], it was found that a considerable (from 1323 to $1379 \mathrm{~K}$ ) spread exists even for the maximum $T_{m}$ values. Therefore accumulation of knowledge of a correlation between the CdTe melt state and crystallization temperature $T_{c r}$ is an urgent problem.

A specific character of CdTe melting (manifestation of an extra endothermic effect (EEE) in melt) has been first demonstrated in [9] using precise differential thermal analysis (DTA). This fact, however, has not received proper attention. More recently, using DTA, for bulky (40 to $100 \mathrm{~g}$ ) CdTe samples, it was found [10-12] that the EEE temperature $T_{\mathrm{EEE}}=T_{m}+10 \mathrm{~K}=1373 \mathrm{~K}$ is the same as a crytical temperature $T^{*}$. The latter is defined in the following way. An ecess over it is accompanied by melt supercooling, $\Delta T^{-}=T_{m}-$ $T_{c r}>0$, while at melt cooling down from $T<T^{*}$ the $\Delta T^{-}=0$. According to [10,11], EEE appears when a bistructural melt (containing microaggregates (clusters) inherited from a crystal) transforms into a single-structural one. DTA of melting made for CdTe samples whose masses ranged from 1 to $5 \mathrm{~g}$ [13] enabled to raise the EEE temperature up to a value of $T_{\mathrm{EEE}}=T_{m} \pm(25-30) \mathrm{K}$. Since in some cases EEE in CdTe melts is of pulsed type [13], it might be of interest to monitor changes in CdTe conductivity in the vicinity of $T_{m}$ in a quasicontinuous mode. The aim of this paper is to study how kinetics of CdTe heating and cooling affects the features of solid-to-liquid and liquid-to-solid phase transitions. For this we used DTA and measurements of melt conductivity at temperatures up to $1473 \mathrm{~K}$.

\section{Experimental}

For our studies we took $p$-CdTe single crystals whose conductivity, $\sigma$, was $10^{-2} \Omega^{-1} \cdot \mathrm{cm}^{-1}$, hole concentration, $p$, was $10^{14} \mathrm{~cm}^{-3}$, and hole mobility at room temperature, $\mu_{298}$, was from 60 to $80 \mathrm{~cm}^{2} / \mathrm{V} \cdot \mathrm{s}$. These crystals have been Bridgmangrown from $\mathrm{Cd}$ and $\mathrm{Te}$ of high purity. After evacuation down to a pressure of $10^{-2} \mathrm{~Pa}$ in ungraphitized quartz ampoules of diameter 8 and height $25 \mathrm{~mm}$, the CdTe blend (mass $0.5 \mathrm{~g}$ ) has been melt. Then a free space over the compact sample was minimized using a quartz plug.

DTA was performed using the following two units: (i) DTA 402 unit (Netsch, Germany), and (ii) a nonstandard modified unit that enables to record time-based thermograms. In the latter case Ar served as both heat-transfer agent and inert atmosphere in the furnace space. In both experimental runs temperature was recorded using $\mathrm{Pt}-\mathrm{Pt} / \mathrm{Rh}$ thermocou- 


\section{L.P. Shcherbak et al: Pre- and postmelting of cadmium telluride}

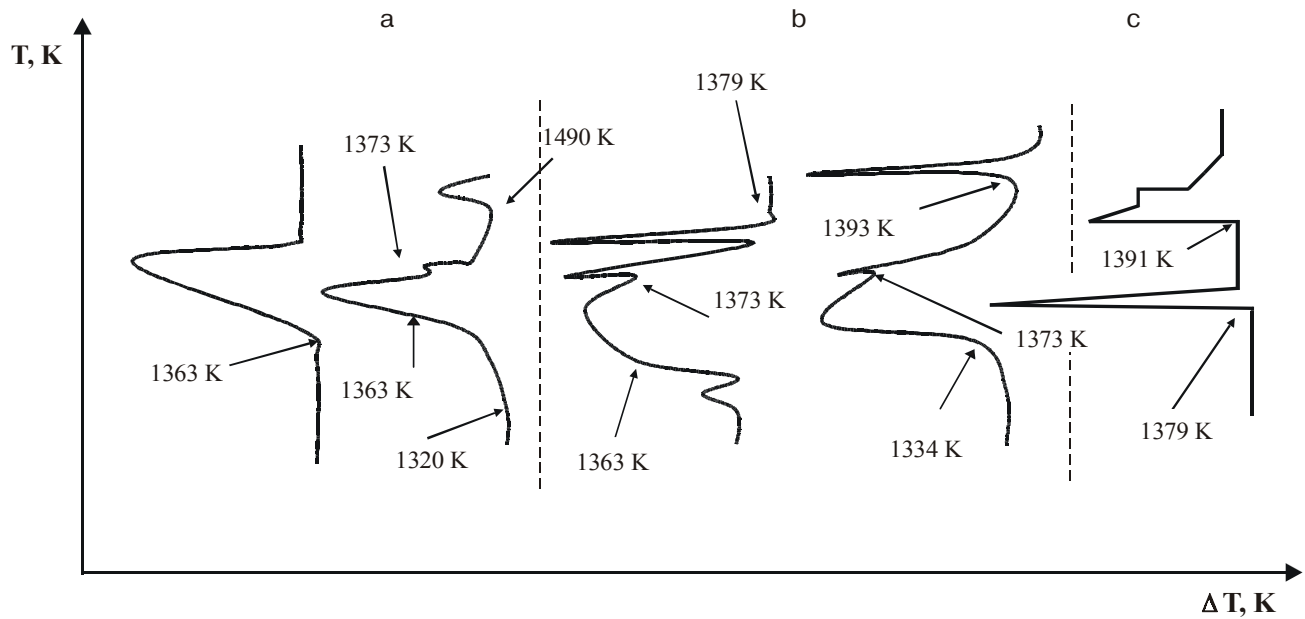

Fig.1. Typical thermograms of CdTe melting: a - heating from temperature $T_{\text {room }}$ up to $1273 \mathrm{~K}$ during 3 min, then heating rate $V_{h}=10$ and $2 \mathrm{~K} / \mathrm{min}$, respectively; b - temperature cycling in the 1420 to $1310 \mathrm{~K}$ range at $V_{h / c}=2$ and $10 \mathrm{~K} / \mathrm{min}$, respectively; $\mathrm{c}-$ heating from $T_{\text {room }}$ at $V_{h}<1 \mathrm{~K} / \mathrm{min}$.

ples that provided an accuracy of $\pm 1 \mathrm{~K}$. The heating $\left(V_{h}\right)$ and cooling $\left(V_{c}\right)$ rates were controlled within 1 to $10 \mathrm{~K} / \mathrm{min}$ range.

The high-temperature conductivity of melts was measured using a four-probe technique in $\mathrm{BN}$ cells; the argon pressure, $P_{\mathrm{Ar}}$, was $13.2 \mathrm{MPa}$. After heating the sample studied (whose mass was $3 \mathrm{~g}$ ) up to $1473 \mathrm{~K}$ we recorded the change in conductivity of the melt that was cooled with a rate of 0.5 to $1 \mathrm{~K} / \mathrm{min}$. After the sample has been cooled $50 \mathrm{~K}$ below the crystallization temperature, it was reheated (with the same rate) up to $1473 \mathrm{~K}$; its conductivity, $\sigma(T)$, was checked at time intervals of $1 \mathrm{~min}$.

\section{Results and discussion}

\subsection{Differential thermal analysis}

After performing a run of experiments for the same sample we have concluded that both heating rate and «previous thermal history» of a sample affect the parameters of solidto-liquid and liquid-to-solid phase transitions in CdTe. At rapid (during $3 \mathrm{~min}$ ) sample heating from room temperature up to $1273 \mathrm{~K}$ followed by heating with a rate of $10 \mathrm{~K} / \mathrm{min}$ a differential thermocouple notes only one abrupt kink of the zero line. This kink is related to the endothermic effect of melting at a melting temperature value $T_{m}(\mathrm{CdTe})=1363 \pm 2 \mathrm{~K}$ that is given in a number of handbooks. At slower ( $V_{h}=2 \mathrm{~K} / \mathrm{min}$ ) heating from $1273 \mathrm{~K}$, however, the readings of a differential thermocouple begin to depart from the datum line at temperatures that are 10 to $50 \mathrm{~K}$ below that at which the endothermic effect of melting at $1363 \pm 2 \mathrm{~K}$ is recorded. In this case a weak EEE appears in a melt at $1373 \mathrm{~K}$ (Fig. 1, a).

The features of CdTe mealting are considerably changed when performing heating-cooling cycles (temperature cycling) in the $1420-1310 \mathrm{~K}$ range and keeping the melt at $1420 \mathrm{~K}$ for $10-30 \mathrm{~min}$. At early stages of temperature cy- cling with $V_{h / c}=10 \mathrm{~K} / \mathrm{min}$ only EEE1 at $1373 \pm 1 \mathrm{~K}$ is observed. Then, at further melting processes EEE2 and EEE3 appear at $1379 \pm 1 \mathrm{~K}$ and $1391 \pm 1 \mathrm{~K}$, respectively (Fig. 1, b). Besides, in a number of cases EEE at $1413 \pm 1 \mathrm{~K}$ is registered. Temperature cycling with a rate $V_{h / c}=2 \mathrm{~K} / \mathrm{min}$ is accompanied with appearance of EEE2 and EEE3 even at the first repetition of melting. At further stages both EEE1 and EEE2 enthalpies increase. This fact is reflected in an increase of the corresponding EEE surface in the thermogram. Thus a character of postmelting that is observed in CdTe melts is controlled with the crystal heating kinetics. At a definite state of crystal defects it occurs not by a single, but by several stages.

By varying kinetics of CdTe sample heating one can obtain one more, unexpected effect. At slow $\left(V_{h}<1 \mathrm{~K} / \mathrm{min}\right)$ heating from room temperature or at multiple temperature cycling in the high-temperature region the sample mealting temperature, $T_{m}$, increases up to $T$ a value of $1379 \pm 1 \mathrm{~K}$ that coincides with that of EEE2. In this case only EEE3 is observed. It is of a pronounced pulsed character (Fig. 1,c). So a spread of data on CdTe $T_{m}$ values that exists in the literature [8] may result from the fact that the samples studied by different authors were of different defect contents.

Judging from the results of investigation of correlations between overheating $\left({ }^{-} T^{+}=T_{\text {melt }}^{\max }-T_{m}\right)$ and cooling $\Delta T^{-}$ of a melt after keeping it at a maximum temperature for $10 \mathrm{~min}$ (Fig. 2, a), its «thermal past history» affects also the crystallization parameters. Contrary to the data given in $[5,6]$, the initial portion of the curve shown in Fig. 2, a at $T<T_{m}+10 \mathrm{~K}$ is characterized by negative $\Delta T^{-}$values, i.e. a melt is spontaneously crystallized at a temperature exceeding $T_{m}$. In this case the $\Delta T^{-}$versus $\Delta T^{+}$curve passes through a minimum. The fact that the crystallization temperature of a material is over $T_{m}$ is an evidence that some structural units that decrease the activation energy of nucleation have retained in a melt. They seem to be crystal-like lattice fragments (clusters) that retain in a melt up to $T_{m}+10 \mathrm{~K}$. The same conclusion can be drawn from an analysis of how the surface of the 


\section{L.P. Shcherbak et al: Pre- and postmelting of cadmium telluride}
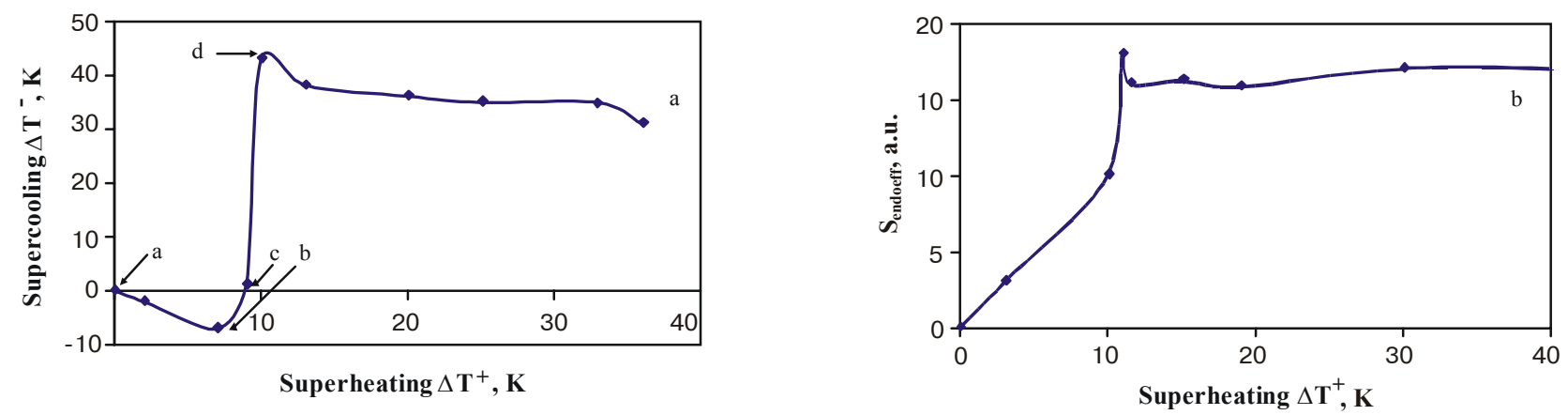

Fig.2. Supercooling $\Delta T^{-}$(a) and endothermic effect area (b) versus CdTe melt superheating curves.

melting heat effect, $S_{\text {endoeff }}$ (that is registered in a thermogram when the melt temperature is kept constant after it has reached $T_{\text {melt }}=T_{m}+\Delta T$, Fig. 2, b) depends on melt superheating. From Fig. 2,b it follows that $S_{\text {endoeff }}$ and, accordingly, the melting enthalpy, peak (at a given heating rate) only when having reached $T_{\text {melt }}=T_{m}+10 \mathrm{~K}$. Further increasing of melt superheating does not change both $S_{\text {endoeff }}$ and melting enthalpy values.

\subsection{High-temperature conductivity of CdTe}

The temperature dependence of CdTe melt conductivity for the case of $V_{h / c}=1 \mathrm{~K} / \mathrm{min}$ is given in Fig. 3. Unlike the $y(T)$ curve at cooling that is monotonous (close to exponential) see the inset in Fig. 3, the $y(T)$ curve taken at heating has a number of kinks at temperatures coinciding with those of EEE1 - EEE3. However, no conductivity jumps were observed. For the sake of simplicity the experimental data on $y$ drop at melt cooling down from $1473 \mathrm{~K}$ are shown in Fig. 3 near $T_{m}$ only. Here, as in [7], the melt conductivity hysteresis is most pronounced. This indicates at supercooling of a highly superheated melt. Shown in Fig. 3 are also the data on $y(T)$ for a CdTe melt whose composition differs from that studied by us (one can see this from the lower value of $T_{m}$ ). However, as may be seen from Fig.3, at temperatures over $1410 \mathrm{~K}$ both $y(T)$ curves completely coincide. This fact may point to stability of content and/or structure of the melt structural units over the temperature range studied, what- ever the starting composition of the crystal and heating kinetics.

It should be noted that according to Ioffe's empirical rule [14], a melt retain semiconducting features $(d y / d T>0)$ only if a short-range order (inherent in a crystalline phase) holds. For CdTe melt one should conclude that orientation of covalent bonding between the crystal components retains up to $1473 \mathrm{~K}$ (though, probably, it is predominantly associative).

\subsection{Premelting}

Since EEE2 and EEE3 are observable only after high-temperature treatment of the solid phase, the reasons for their appearance may be premelting effects. This statement implies usually processes that are related to an intense production of point and linear defects. At a definite defect content in a crystal the temperature dependencies of a number of structural-sensitive parameters (specific enthalpy, specific heat $C_{p}$, molar volume, etc.) demonstrate extrema. Their presence is viewed as a proof of the premelting effects that are, on frequent occasion, related to jump-type changes in crystal structure $[1,2]$.

The temperature dependence of $C_{p}$ for CdTe based on a totality of existing literature data may be approximated by two lines that are almost parallel. This does not form the basis for a conclusion that an extremum exists near $T_{m}$. However, a departure of the datum line immediately before $T_{m}$ (that is observed when performing temperature cycling) may

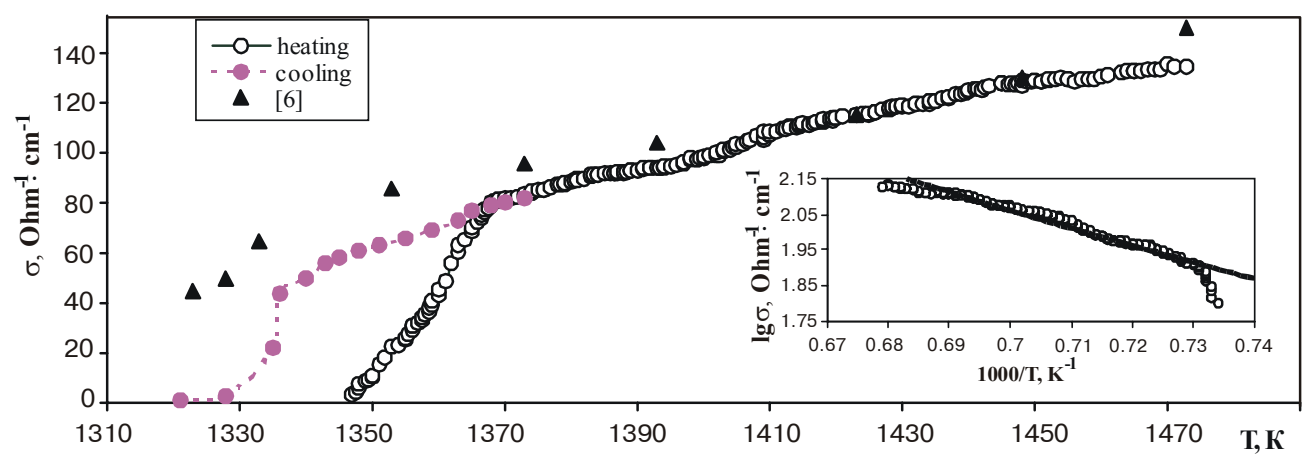

Fig. 3. Temperature dependence of CdTe conductivity at CdTe melt heating and cooling; $\boldsymbol{\Delta}$ - data of [6]. 


\section{L.P. Shcherbak et al: Pre- and postmelting of cadmium telluride}

be attributed to a change in the $C_{p}(T)$ curve slope due to an intense defect production in a crystal. Indeed, according to [16] at $T \geq T_{m}-50 \mathrm{~K}$ congruent sublimation in the CdTe lattice is enchanced. Due to a growth of the point defect (vacancies in both sublattices) content in a crystal, some prerequisites to the structural reconstruction (either local or over the whole volume) appear. There exist some inferences in the literature about occurrence of one or several polymorphic transformations in the CdTe lattice. These inferences were made basing on circumstantial evidence on the temperature dependence of a number of structural-sensitive properties [17]. But experimental determination of a nature of structural transitions in CdTe made by using direct diffraction techniques is hindered due to component volatility and chemical aggressiveness. At the same time a considerable $T_{m}$ increase after the sample has been thermally treated at premelting temperatures for a rather long time (the effect observed in the present paper) serves as a weighty circumstantial evidence that a structural transformation has occurred in the crystal studied. Taking into account an occurrence of a polymorphic modifications in the crystal lattices of II - VI compounds [18], one can assume that the same process occurs also in the lattice of CdTe at high temperatures. Indeed, according to [19], a hexagonal modification was recorded, at room temperature, in the CdTe crystal that was quenched after long-term keeping at $1323 \mathrm{~K}$.

\subsection{Postmelting}

As was shown in [20], the CdTe molar volume $V_{m}$ changes under melting slightly $\left(\Delta V_{m}=0.6 \%\right)$. This correlates with the evidence (given in Fig. 2, b) that melting is not completed at $\mathrm{T}_{m}$. Since the enthalpy of CdTe melting peaks only at $T_{m}+10 \mathrm{~K}$, one should consider that below that temperature some rather big fragments are present in the melt. They inherit the crystal lattice structure. Different terms (grains, aggregates, conglomerates) are used in the literature to denote such fragments. This fact characterizes most likely distinctions in size for these particles, as well as their ability to grow or disintegrate, depending on thermal fluctuations in a melt.

It is most convenient to explain the superheating-supercooling correlations (presented in Fig. 2, a) in terms of the cluster model for melting [21]. Within this model a transformation of a two-structure melt with crystal-like clusters to a uniform medium may occur in two ways: through fragmentation (breaking up) or dissolving individual surface atoms (defect drain). In the first case the cluster sizes decrease, while the cluster content grows. Dissolution from the surface is accompanied by a change of cluster content that occurs in agreement with their size decreasing. According to Fig. 2,a the fragmentation mechanism is predominant near $T_{m}$ and an excess of $T_{\text {cryst }}$ over $T_{m}$ is related to a presence in a melt of many retained crystallization centers. Over the temperature corresponding to the minimum on the $\Delta T^{2}=$ $f\left(\Delta T^{+}\right)$curve the defect drain mechanism becomes predominant, and content of existing crystallization centers goes down. Thus presence of a minimum near $T_{m}$ (Fig. 2, a) indi- cates at a change of the mechanism of structural transformations in a melt under the heating conditions used. Following the line of these considerations one has to assume that at the inflection point on the $\Delta T^{2}=f\left(\Delta T^{+}\right)$curve (that coincides with the EEE temperature) the existing clusters or smaller structural units (say, tetrahedrons) still serve as the equilibrium crystallization centers during further cooling. At $T>T$ (EEE1) such nuclei are absent or their mutual coordination becomes unfavorable for further size growth, and this results in melt supercooling.

The appearance of EEE2 and EEE3 in the CdTe melt may be explained as a result of interrelation between pre- and postmelting. On the assumption of local formation of the wurtzite phase under high-temperature treatment of the crystal, EEE2 may be related to a dissociation of the 6-link rings into chains; breaking of chains manifests itself in thermograms as EEE3. It is likely that a substantial (up to $1379 \mathrm{~K}$ ) increase in the sample melting temperature that is observed at low heating rate corresponds to an increase of the high-temperature phase (wurtzite) fraction or to a complete transformation of the whole volume into a high-temperature modification.

\section{Conclusions}

Cadmium telluride melting proceeds nonisothermically and is accompanied with only partial structural modification at $T_{m}$. Further increase of the melt temperature is accompanied by a structural transformation that occurs by stages. Every melting stage has its own supercooling depending on the value of melt superheating over $T_{m}$. In the premelting temperature range polymorphic transformations may occur. They lead to formation of a phase that is melting more difficultly. This results in $T_{m}$ increase from 1365 to $1379 \pm 1 \mathrm{~K}$.

\section{References}

1. A.R.Ubbelode. Melted State of Matter (Russian translation), Metallurgiya, Moscow (1982).

2. W.Hayes. Premelting // Contemp.Phys. 27(6), pp.519-532 (1986).

3. L.A.Bityutskaya, E.S.Mashkina. Features of pre-and posttransition states at copper melting (in Russian) // Pisma v ZhTF, 21(24), pp.90-93 (1995).

4. L.A. Bityutskaya, E.S.Mashkina. Cooperative effects of pre- and posttransition states at germanium melting (in Russian) // Pisma v ZhTF, 21(18), pp.8-11 (1995).

5. H.J.Koh, P.Rudolph, N.Shafer, K.Umetsu, T.Fukuda. The effect of various thermal treatments on supercooling of PbTe melts // Mater. Sci. Engin. B 34, pp.199-203 (1995).

6. V.M.Glazov, S.N.Chizhevskaya, N.N.Glagoleva. Liquid Semiconductors (in Russian). Nauka, Moscow (1967).

7. Yu.V.Rud', N.V.Sanin. Conductivity of cadmium telluride in the vicinity of melting temperature (in Russian) // Fiz. Tekhn. Poluprov. 5(8), pp.1587-1595 (1971).

8. N.J. Tianrong, A. Silk, A.W. Watson et al. Thermodynamic and phase diagram assessment of the $\mathrm{Cd}-\mathrm{Te}$ and $\mathrm{Hg}$-Te systems // CALPHAD, (3), pp. 399-414 (1995).

9. H.Shamsuddin, A.Nazar. Thermodynamic properties of cadmium telluride // High Temp. Sci., 28, pp. 245-254 (1990).

10. P.Rudolph, M.Mülberg. Basic problem of vertical Bridgman growth of CdTe // Mater. Sci. Engin., B16, pp. 8-16 (1993). 


\section{L.P. Shcherbak et al: Pre- and postmelting of cadmium telluride}

11. M.Mülberg, P.Rudolph, M.Laasch. Correlation between supercooling and superheating in CdTe during unseeded Bridgman growth // $\mathrm{J}$. Cryst. Growth, 128, pp. 571-575 (1993).

12. P.Rudolph. Fundamental studies of Bridgman growth of CdTe // Progr. Crystal Growth and Charact., 29, pp. 275-281 (1994).

13. O.Panchouk, L.Shcherbak, P.Feichouk, Effect of CdTe «postmelting» // J. Cryst. Growth, 161, pp. 16-19 (1996).

14. A.Ioffe, F.Regel. Progress in Semiconductors (London) 4, p.237 (1960).

15. H.Maleki and L.R.Holand. Thermal properties of CdZnTeSe compounds // In: Properties of Narrow-gap Cadmium-based Compounds. Ed. by Capper. GEC-Marconi Infra-Red Limited. London. UK (1994).

16. Ya.Kh.Grinberg, V.N.Gus'kov, V.B.Lazarev, M.Ya. Zel'venskii. $p-T-x$ phase equilibria in the Cd-Te system (in Russian) // Neorgan. Mater., 21(12), pp. 1991-2004 (1989).
17. Yu. M.Ivanov. Growth and homogeneity region of CdTe // J.Cryst. Growth, 161, pp.12-15 (1996).

18. Physics and Chemistry of II-VI Compounds, ed. by M.Aven and J.S.Prener. North-Holland Publ. Co., Amsterdam (1967).

19. Yu.P.Gnatenko, M.V.Kurik, V.V.Matlak. Band structure of hexagonal CdTe //Phys. Letters, 29, pp. 522-523 (1969).

20. N.N.Kolesnikov, M.P.Kulakov, Yu.N.Ivanov. Some properties of melts of $\mathrm{A}^{2} \mathrm{~B}^{6}$ compounds // J.Cryst. Growth, 125, pp. 576582 (1992).

21. V.M.Glazov. Present development of investigations of the postmelting effect in semiconductor melts (in Russian) // Neorgan. Mater. 31 (II), pp. 1287-1305 (1996). 